neighbouring Bulgaria, which shares a similar history — have emigrated as soon as they could. Very few have returned. It remains hard to do science in these countries, even though both joined the European Union in 2007. A few bright spots exist, but too much of the research landscape is still dominated by old-guard scientists who don't produce results, resist the introduction of international research standards and block the system to fresh blood.

As we report on page 142, the tide may just have turned for Romanian scientists. The government there is boosting funds and seems to know what is required for them to be spent wisely, and how to overcome scepticism among research émigrés. The Romanian government has a serious long-term plan for science, and this deserves recognition. Romanian scientists abroad, Giosan among them, are starting to smile.

The sentiment is not shared by those who watch the situation in Bulgaria with mounting despair. The Bulgarian government has only a short-term plan, the long-term consequences of which are likely to be disastrous. Funds have been slashed and the control of dozens of research institutes is set to be handed to the government from the Bulgarian Academy of Sciences, which will survive only as an academy. To separate active research from a learned society is not necessarily wrong - the status of both Britain's Royal Society and France's Académie Française demonstrates that — but the Bulgarian government is yet to show that it knows what to do with the institutes it is so keen to adopt.

In fact, it is clear that this populist government - which took office in July 2009 on an anti-corruption mandate - is not interested in science, and has convinced many among the general public that it is a waste of

money. Its science and education minister, Sergei Ignatov, was politically too weak to oppose a budget cut of more than one-third ordered in mid2010. Science in Bulgaria has been humiliated as never before.

It is true that the Bulgarian Academy of Sciences needs deeper reform.

"A poorly performing science base cannot be fixed by just throwing money at it."
Under pressure from previous governments to raise its game, the academy organized an international evaluation and slimmed down to a fraction of the size it was in richer, Soviet times. But greater change is needed.

As the Romanian government has noted, a poorly performing science base cannot be fixed by just throwing money at it: regulations need to ensure that the money is well used. But in the same way, reforms are pointless if budgets are so restricted that little serious research can be performed - as is now the case in Bulgaria.

The Bulgarian government, together with its scientists, must urgently create a long-term scientific plan for the country, and a strategy to put the plan into operation and ensure that it is successful. It cannot afford to reject the European Union philosophy of a future centred on a knowledge-based economy.

In the meantime, it needs to restore budgets for science and universities to levels that allow them to function properly, and delay plans to break up the academy. Only when a proper long-term strategy is in place will the government know what it needs to do about its research institutes and their budgets. If it needs inspiration in this, it need only look north to its neighbour Romania. The contrast between the age of wisdom and the age of foolishness is clear.

\section{First do no harm}

\section{Simple tools to diagnose mental illness should not be offered without sound supporting evidence.}

\section{I} ncorrect diagnosis of people with psychiatric disorders has farreaching implications. Miss the manic phases of people with bipolar disorder, for instance, and, rather than the mood-stabilizers they need, they might be given antidepressants. The drugs could make them 'hypomanic' - a state in which they might spend money recklessly, invest irrationally and jabber incessantly so that friends and employers no longer want them around - and tip them into even more extreme bipolar cycles. Meanwhile, if the hallucinations of a person with schizophrenia don't become apparent during analysis, the patient may likewise be diagnosed as depressed, be given antidepressants and go on to become even more withdrawn. Unfortunately, misdiagnosis happens all too often - in around $70 \%$ of cases of bipolar disorder, according to some estimates. And such mistakes often go uncorrected for years.

The problem is that, in the absence of clear-cut biological markers for such disorders, doctors depend on subjective examinations to fit patients into poorly defined categories. The uncertainty inherent in these diagnoses is a menace, and not only for patients. The problem affects pharmaceutical companies working to develop new drugs, insurance companies trying to determine coverage, health-care systems and employers. Researchers are desperate for objective diagnostic markers to replace subjective examinations. In their search they have studied genetics and investigated tools such as electroencephalography and functional magnetic resonance imaging. Despite many claims and limited successes, so far none of these findings has been replicated consistently enough to merit widespread clinical use.

Last year, doctors in Japan started using a relatively new technique - near-infrared spectroscopy (NIRS) - to distinguish between schizophrenia, depression, bipolar disorder and normal mental-health states (see page 148). It is not difficult to see why this was a popular move. Doctors like it because it is easy to use. Patients like its objective nature, and that it provides them with physiological evidence of a disorder. And officials at the health ministry are happy because it represents a public success of their drive to innovate.

In Japan, NIRS diagnosis is one of dozens of advanced medical techniques offered to patients - at their own expense - despite not having gone through the clinical trials needed for approval by national health insurance. But is it ready for the clinic? Most scientists contacted by Nature do not think so. The patient groups of the supporting studies were small. The tests have not been reproduced in various clinical settings as one might hope. There is no international consensus on how best to measure NIRS parameters, much less a clear consensus on how to apply them to mental health. And if it is not ready, the same advantage that has patients lining up for it - the authority of an objective, physiological measure, the air of the incontrovertible - will become an obstacle. A misdiagnosis that carries the authority of an 'objective' measurement will probably be even more difficult to overturn.

The doctors who offer the technique say that it is only an aid, just one tool in their toolbox. Indeed, they are following Japan's advanced medical technology protocol properly and offering, in good faith, a diagnostic test that they believe works. Their attempts to use it seem sincere, and not motivated by profit. Still, if the technique has not been clinically validated, patients should not be paying for it, particularly given the challenging scientific landscape of mental-health problems. Japan's advanced medical-technologies programme is blurring the line between protocols that have been properly validated and those that have not. More rigorous verification methods are needed, starting with multiple blind trials in large patient groups.

NIRS does show promise. It is easy, quick and, perhaps combined with other diagnostic techniques, could be a powerful tool, if the right validation studies are done. If the clinics that offer the technique are so sure that it works, then they should present the supporting evidence to prove it.
SNATURE.COM To comment online, click on Editorials at: go.nature.com/xhunqv
Meanwhile, other neuroscientists should continue the search for more conventional biomarkers. To offer better care to people with mental-health problems is a noble motive, but one that needs to have science alongside desire at its heart. 